genes expand.

Understanding

which mutations are

present in these smaller

groups of cells could aid in

the selection of therapies

for individuals.

Sci. Transl. Med. 7, $283 r a 54$

(2015)

PALAEONTOLOGY

\section{Ancient seas bore bone-fed worms}

Bone-eating worms devour dead whales in today's oceans, but their ancient relatives might have emerged millions of years before their modern food source.

Modern Osedax worms drill distinctive holes in bone, with the oldest examples found in whale and fish bones from around 30 million years ago. But Silvia Danise and Nicholas Higgs of Plymouth University, UK, found these boreholes in the fossilized remains of an extinct marine reptile and a turtle, both roughly 100 million years old.

The discovery shifts the evolution of Osedax-type worms to well before the rise of early whales, about 50 million years ago. The ancient worms probably survived on the bones of turtles and other animals before whales appeared.

Biol. Lett. 11, 20150072 (2015)

\section{ECOLOGY}

\section{Like a moth to a trumpet flower}

Hawk-moths are better at finding nectar in flowers shaped like the bell of a trumpet than in those that resemble a flat disc.

Eric Octavio Campos and his colleagues at the University of Washington in Seattle used a $3 \mathrm{D}$ printer to create flowers

\section{, \\ ,}

\section{$\sqrt[3]{\text { that were }}$}

either flat

or curved like a

trumpet. The team

inserted a tube filled with

sugar water into the centre of each flower, and allowed hawk-moths (Manduca sexta; pictured) to feed from them. Although the moths visited each type of flower equally, they drained trumpet flowers more often than flat ones. The results suggest that the trumpet shape helps to guide the moth's probing mouthparts to nectar.

Before the advent of $3 \mathrm{D}$ printers, ecologists who studied plant-pollinator interactions had to breed flowers or sculpt fake ones by hand. The 3D-printing technology allowed Campos's team to quantify flower shape and produce precise models with ease.

Funct. Ecol. 29, 462-468 (2015)

ANIMAL BEHAVIOUR

\section{Octopus crawls with no rhythm}

Octopuses can move quickly in any direction, regardless of which way the eyes and body are facing.

Binyamin Hochner of the Hebrew University in Jerusalem and his colleagues studied the animal's movement by analysing videos of crawling octopuses (Octopus vulgaris; pictured). They found that the radially symmetrical octopus moves by extending and shortening its arms, and that it tends to crawl diagonally relative to its line of sight.

The octopus also seems to lack any rhythmic pattern when coordinating the movement of its arms, unlike most other animals. The authors suggest that octopuses make last-minute decisions
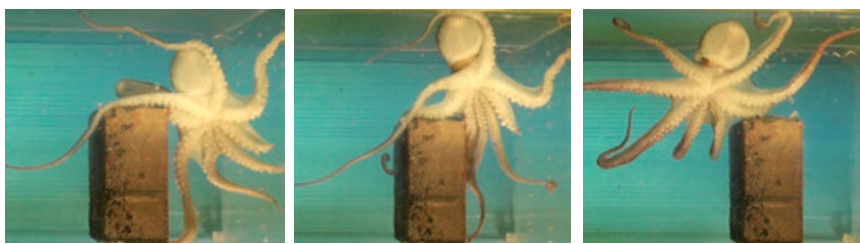

about which arm to move to determine their direction of crawling.

Curr. Biol. http://doi.org/3vd (2015)

\section{ASTROPHYSICS}

\section{Many flavours of supernova}

Exploding stars grouped in one family because of their similarities actually form two distinct groups. This may have important cosmic implications because the explosions, called supernovae, are the primary evidence that the Universe's expansion is accelerating.

Half of type Ia supernovae seem to have similar intrinsic brightnesses when seen in the visible spectrum. But when Peter Milne of the University of Arizona in Tucson and his team analysed data from the Hubble Space Telescope and NASA's Swift satellite, they found that the supernovae fell into two subfamilies, each brighter than the other in a different part of the ultraviolet spectrum.

The relative abundances of the two subfamilies seem to have changed over the past several billion years, a fact that could complicate their use as markers of cosmic expansion, the authors say.

Astrophys. J. 803, 20 (2015)

\section{ANIMAL BEHAVIOUR}

\section{Dazzling colours distract predators}

Many animals have coloration that shifts depending on the angle from which they are viewed, and this may help them to avoid predators.

This 'interference coloration' has evolved several times in beetles, birds, fish and other creatures, but it is not clear why. Thomas Pike, now at the University of Lincoln, UK, trained laboratory Japanese quails (Coturnix japonica) to peck at moving targets on a screen. When the targets changed from green to blue as they moved across the screen, the quails required many more pecking attempts to successfully 'capture' the mock prey. They were also less accurate with their pecks than when the targets stayed the same colour.

The findings suggest that this kind of coloration impairs a predator's ability to accurately locate prey.

Biol. Lett. 11, 20150159 (2015)

\section{ONATURE.COM}

For the latest research published by Naturevisit:

www.nature.com/latestresearch 